\title{
Impact of tumor characteristics and pre- and postoperative hormone levels on hormonal remission following endoscopic transsphenoidal surgery in patients with acromegaly
}

\author{
Tyler Cardinal, BS, ${ }^{1}$ Martin J. Rutkowski, MD, ${ }^{1}$ Alexander Micko, MD, PhD,, ${ }^{1,2}$ Mark Shiroishi, MD, ${ }^{3}$ \\ Chia-Shang Jason Liu, MD, PhD, ${ }^{3}$ Bozena Wrobel, MD, ${ }^{5}$ John Carmichael, MD, ${ }^{1,4}$ and \\ Gabriel Zada, MD'
}

\begin{abstract}
1Department of Neurosurgery, USC Pituitary Center, Keck School of Medicine of University of Southern California, LoS Angeles, California; ${ }^{2}$ Department of Neurosurgery, Medical University of Vienna, Austria; ${ }^{3}$ Department of Radiology, Division of Neuroradiology, Keck School of Medicine of University of Southern California; ${ }^{4}$ Department of Medicine, Division of Endocrinology and Diabetes, Keck School of Medicine of University of Southern California; and ${ }^{5}$ Department of Otolaryngology, Keck School of Medicine of University of Southern California, Los Angeles, California
\end{abstract}

OBJECTIVE Acromegaly is a disease of acral enlargement and elevated serum levels of insulin-like growth factor-1 (IGF-1) and growth hormone (GH), usually caused by a pituitary adenoma. A lack of consensus on factors that reliably predict outcomes in acromegalic patients following endoscopic endonasal transsphenoidal surgery (EETS) warrants additional investigation.

METHODS The authors identified 52 patients with acromegaly who underwent an endoscopic endonasal approach (EEA) for resection of a $\mathrm{GH}$-secreting pituitary adenoma. Preoperative and postoperative tumor and endocrinological characteristics such as tumor size, invasiveness, and GH/IGF-1 levels were evaluated as potential indicators of postoperative hormonal remission. Endocrinological remission was defined as postoperative IGF-1 levels at or below the ageand sex-normalized values.

RESULTS The 52 patients had a mean age of $50.7 \pm 13.4$ years and a mean follow-up duration of $24.4 \pm 19.1$ months. Ten patients (19\%) had microadenomas and $42(81 \%)$ had macroadenomas. Five patients $(9.6 \%)$ had giant adenomas. Forty-four tumors (85\%) had extrasellar extension, with 40 (77\%) exhibiting infrasellar invasion, 18 (35\%) extending above the sella, and $7(13 \%)$ invading the cavernous sinuses. Thirty-six patients (69\%) underwent gross-total resection (GTR; mean maximal tumor diameter $1.47 \mathrm{~cm}$ ), and $16(31 \%)$ underwent subtotal resection (STR; mean maximal tumor diameter $2.74 \mathrm{~cm}$ ). Invasive tumors were significantly larger, and Knosp scores were negatively correlated with GTR. Thirty-eight patients (73\%) achieved hormonal remission after EEA resection alone, which increased to $87 \%$ with adjunctive medical therapy. Ninety percent of patients with microadenomas and $86 \%$ of patients with macroadenomas achieved hormonal remission. Preoperative IGF-1 and postoperative day 1 (POD1) GH levels were inversely correlated with hormonal remission. Postoperative CSF leakage occurred in 2 patients (4\%), and none experienced vision loss, death, or injury to internal carotid arteries or cranial nerves.

CONCLUSIONS Endoscopic transsphenoidal resection of $\mathrm{GH}$-secreting pituitary adenomas is a safe and highly effective treatment for achieving hormonal remission and tumor control in up to $87 \%$ of patients with acromegaly when combined with postoperative medical therapy. Patients with lower preoperative IGF-1 and POD1 GH levels, with less invasive pituitary adenomas, and who undergo GTR are more likely to achieve postoperative biochemical remission.

https://thejns.org/doi/abs/10.3171/2020.3.FOCUS2080

KEYWORDS pituitary; adenoma; acromegaly; somatotroph; cavernous sinus; invasion; surgery; Knosp; endoscope

ABBREVIATIONS DI = diabetes insipidus; EEA = endoscopic endonasal approach; EETS = endoscopic endonasal transsphenoidal surgery; EOR = extent of resection; GH = growth hormone; GTR = gross-total resection; IGF-1 = insulin-like growth factor-1; IHC = immunohistochemistry; LAC + USC = Los Angeles County + University of Southern California; OGTT = oral glucose tolerance test; POD1 = postoperative day $1 ; \mathrm{SRL}=$ somatostatin receptor ligand; $\mathrm{STR}=$ subtotal resection; $\mathrm{TSH}=$ thyroid-stimulating hormone.

SUBMITTED January 30, 2020. ACCEPTED March 11, 2020.

INCLUDE WHEN CITING DOI: 10.3171/2020.3.FOCUS2080. 
$\mathrm{A}$ CROMEGALY is a disease of excess growth hormone (GH) secretion usually due to a $\mathrm{GH}$-secreting pituitary adenoma. ${ }^{14,19}$ It is characterized by bony and soft-tissue enlargement, and a host of comorbidities, due to elevated levels of insulin-like growth factor-1 (IGF-1) and GH. ${ }^{13,14}$ Treatment is critical as normalization of $\mathrm{GH}$ levels significantly reduces comorbidity and mortality rates. ${ }^{13,14}$ Acromegaly is considered in remission when age- and sexnormalized IGF-1 levels fall within normal limits, when random $\mathrm{GH}$ levels are less than $1 \mu \mathrm{g} / \mathrm{L}$, or when a nadir $\mathrm{GH}$ of less than $0.4 \mu \mathrm{g} / \mathrm{L}$ is obtained after oral glucose tolerance test (OGTT). ${ }^{7,15}$

Resection alone has been shown to control disease in up to $88 \%$ of microadenoma cases and $50 \%$ of macroadenoma cases. ${ }^{6}$ While resection remains the primary treatment for acromegaly, somatostatin receptor ligands (SRLs), GH receptor antagonists, dopamine agonists, and radiotherapy offer further means of achieving biochemical remission and tumor control. ${ }^{13,14}$

Numerous studies have investigated predictors of postoperative outcomes in patients with acromegaly who undergo endoscopic endonasal transsphenoidal surgery (EETS) for their GH-secreting pituitary adenomas. ${ }^{1-4,6,8-10}$, $12,16,18,21,23-28$ Tumor size has been shown to be predictive of disease remission, with patients harboring smaller tumors more likely to undergo postoperative remission. ${ }^{6,11,21}$ Adenomas that exhibit parasellar or infrasellar invasion have been repeatedly shown to be independently associated with lower rates of postoperative cure. ${ }^{12,24,27}$ Other studies have correlated preoperative $\mathrm{GH}$ levels, ${ }^{1,6}$ postoperative $\mathrm{GH}$ levels, ${ }^{12,24}$ and hormonal remission. Sex,${ }^{25}$ history of prior surgery, ${ }^{27}$ and preoperative absence of pituitary deficit $^{1}$ have also all been found to be predictive of postoperative cure. The significance of pre- and immediate postoperative IGF-1 levels remains undetermined.

In the following study, we analyze information obtained from 52 acromegaly patients who underwent EETS between September 2012 and June 2019. Here we study the relationships among biochemical remission, tumor control, and preoperative size, pattern of invasion, and levels of GH and IGF-1.

\section{Methods}

\section{Patient Cohort and Evaluation}

We retrospectively reviewed our database of surgeries performed by the senior author (G.Z.) at Los Angeles County + University of Southern California (LAC + USC) Medical Center and Keck Hospital of USC between September 2012 and July 2019. Sixty-nine patients who underwent EETS for resection of suspected GH-secreting pituitary adenomas were identified, with 3 patients requiring an extended approach. Seventeen patients without follow-up MRI or IGF-1 levels were later excluded, leaving 52 patients for final analysis. For each surgery, the pathology, type of exposure, presence of an intraoperative CSF leak, tumor consistency, and complications were recorded. Based on Schroeder and colleagues' conclusion that a small percentage of tumors from clinically acromegalic patients do not stain positive for $\mathrm{GH}$ on immunohistochemistry (IHC), 1 patient without a pathologically confirmed $\mathrm{GH}$-secreting tumor but with significantly elevated preoperative IGF-1 levels and acromegalic signs and symptoms was included. ${ }^{22}$ The institutional review board at the Keck School of Medicine approved the study.

\section{Radiological Evaluation}

All patients underwent preoperative MRI to assess tumor size, presence of cavernous sinus invasion, infrasellar invasion, suprasellar extension, sphenoid anatomy, carotid anatomy, presence of residual pituitary gland, and the position of the optic chiasm and nerves. Patients underwent MRI at 3 months postoperatively to assess extent of resection (EOR), and then annually to monitor tumor progression or recurrence. Tumor size was defined by its maximal diameter in either the anteroposterior, lateral, or craniocaudal dimension. Parasellar extension into the cavernous sinus(es) was assessed according to the Knosp classification, consisting of 4 grades based on proximity to the internal carotid artery. Knosp scoring was performed at weekly clinical conferences and confirmed by a second independent reader (M.S., A.M.) for all available images.

\section{Endocrinological Evaluation}

Levels of IGF-1 were measured preoperatively as part of a comprehensive endocrinological evaluation, including a full pituitary panel. Preoperative $\mathrm{GH}$ levels were not obtained in all patients due to low clinical utility as a result of its pulsatile nature. Postoperative $\mathrm{GH}$ and cortisol levels were measured on the morning of postoperative day 1 (POD1), and follow-up IGF-1 levels were obtained at 6 weeks and 12 weeks postoperatively, in addition to a full pituitary panel to assess for the presence of any new endocrinopathy or hypopituitarism. Biochemical remission was defined as IGF-1 levels at or below the age- and sex-normalized values. OGTT was not performed in every patient but was reserved for equivocal cases with IGF-1 reduction not normalizing or rising during the postoperative timeframe, and when performed was done after 12 weeks postoperatively.

\section{Surgical Technique}

All patients underwent EETS. Briefly, patients were positioned supine with their heads in slight flexion and rotated toward the surgeon to allow for a two-surgeon, fourhanded binostril approach. The right lateral thigh was prepared and draped universally to allow for fat and fascia lata harvest in cases of CSF leak. Following administration of perioperative antibiotics, stereotactic neuronavigation was registered. The endoscope was introduced, the inferior, middle, and superior turbinates were sequentially lateralized, and a posterior septectomy was performed, followed by a wide bilateral sphenoidotomy to allow for a panoramic view of the sella, tuberculum sellae, and lateral sphenoid recesses. The sella was opened widely to each medial cavernous sinus wall, and the dura was opened in an X-shaped fashion following Doppler sonography. Tumors were removed using a combination of extracapsular dissection, where possible, and curettage, including exploration and probing of the medial cavernous sinus walls using angled endoscopy in cases with invasive tumors. 
Following resection, the sellar cavity was packed lightly with hemostatic agents such as Gelfoam and SURGICEL, followed by placement of fat, inlay/onlay fascia lata layers, and fibrin glue in cases of low-grade intraoperative CSF leaks. In patients with high-flow intraoperative CSF leaks, a pedicled nasoseptal flap and temporary lumbar drain prior to extubation are utilized; otherwise, we do not routinely utilize flaps or drains.

\section{Statistical Analysis}

Continuous variables are displayed as the means \pm standard deviations or as medians with interquartile ranges (IQRs) based on normality. Categorical variables are displayed as frequencies. Means were compared using the independent-samples t-test when the variable was normally distributed, or the Mann-Whitney U-test when the distribution was not normal. Categorical variables were analyzed using Pearson's chi-square test or Fisher's exact test. Correlations were determined using Spearman's correlation coefficient test or the Pearson correlation coefficient test. ANOVA with Tamhane's T2 post hoc testing was used to assess the means between 3 or more variables. Statistical analysis was performed using SPSS 24.0 statistical software (IBM Corp.). A 2-tailed p value of $<0.05$ was considered significant.

\section{Results}

\section{Patient Presentation and Clinical Examination}

Fifty-two patients who underwent endoscopic transsphenoidal resection of a suspected GH-secreting pituitary adenoma were included in the full analysis. The group consisted of 16 males (31\%) and $36(69 \%)$ females with a mean age of $50.7 \pm 13.4$ years (Table 1 ). All exhibited signs of acromegaly. Fifty patients $(96 \%)$ presented with dysmorphic craniofacial features, 37 (71\%) with increased ring or feet size, $13(25 \%)$ with skin tags, and $10(19 \%)$ with excessive sweating. Of patients with dysmorphic craniofacial features, 25 (48\%) presented with prognathism, 22 (42\%) with frontal bossing, and 17 (32\%) with macroglossia. Other common presenting symptoms were headache (21 patients, $40 \%$ ), fatigue ( 9 patients, $17 \%$ ), and decreased libido (5 patients, $10 \%$ ). The majority (45 patients, $87 \%$ ) presented with normal neurological examination findings. Four patients (8\%) presented with visual field cuts, 4 (8\%) reported a loss in visual acuity, and 2 (4\%) presented with ophthalmoplegia (Table 1).

Four patients $(8 \%)$ had undergone prior transsphenoidal resection and 1 (2\%) had undergone prior pterional craniotomy. Three of these patients $(6 \%)$ received postoperative adjuvant therapy. One patient $(2 \%)$ received radiographic therapy at an outside hospital and was placed on octreotide. Another patient (2\%) received Gamma Knife radiotherapy and was placed on octreotide. The last patient (2\%) was placed on cabergoline and octreotide dual therapy. Five other patients $(10 \%)$ presented after failed prior medical management. Four patients $(8 \%)$ were prescribed cabergoline, and $1(2 \%)$ was prescribed octreotide in an attempt to control hyperprolactinemia and GH secretion in presumed mammosomatotroph (Table 1). Resection was recommended due to inadequate disease control.
TABLE 1. Patient demographics and preoperative characteristics

\begin{tabular}{|c|c|}
\hline Demographic \& Preop Characteristics & Value \\
\hline \multicolumn{2}{|l|}{ Sex } \\
\hline Male & $16(31)$ \\
\hline Female & $36(69)$ \\
\hline Mean age, yrs & $50.7 \pm 13.4$ \\
\hline \multicolumn{2}{|l|}{ Follow-up, mos } \\
\hline Mean imaging & $21.7 \pm 18.4$ \\
\hline Mean clinical & $24.4 \pm 19.1$ \\
\hline \multicolumn{2}{|l|}{ Hospital } \\
\hline LAC + USC & $18(35)$ \\
\hline Keck & $34(65)$ \\
\hline \multicolumn{2}{|l|}{ Clinical symptoms } \\
\hline Facial changes & $50(96)$ \\
\hline Increased ring/feet size & $37(71)$ \\
\hline Headache & $21(40)$ \\
\hline Arthralgia & $12(23)$ \\
\hline Sweating & $10(19)$ \\
\hline Fatigue & $9(17)$ \\
\hline Decreased libido & $5(10)$ \\
\hline \multicolumn{2}{|l|}{ Clinical signs } \\
\hline Prognathism & $25(48)$ \\
\hline Frontal bossing & $22(42)$ \\
\hline Macroglossia & $17(32)$ \\
\hline Skin tags & $13(25)$ \\
\hline Tooth gaps & $9(17)$ \\
\hline Acanthosis nigricans & $5(10)$ \\
\hline Sleep apnea & $5(10)$ \\
\hline Oily/thickened skin & $3(6)$ \\
\hline Carpal tunnel disease & $2(4)$ \\
\hline \multicolumn{2}{|l|}{ Preop exam findings } \\
\hline Visual acuity loss & $4(8)$ \\
\hline Visual field cuts & $4(8)$ \\
\hline Ophthalmoplegia & $2(4)$ \\
\hline No deficits & $45(87)$ \\
\hline \multicolumn{2}{|l|}{ Prior treatment } \\
\hline Transsphenoidal surgery & $4(8)$ \\
\hline Craniotomy & $1(2)$ \\
\hline Postop adjuvant therapy & $3(6)$ \\
\hline Cabergoline monotherapy & $4(7)$ \\
\hline Octreotide monotherapy & $1(2)$ \\
\hline None & $41(79)$ \\
\hline \multicolumn{2}{|l|}{ Laboratory values (IQR), ng/ml } \\
\hline Median preop GH & $11.1(3.2-37.1)$ \\
\hline Median preop IGF-1 & $576(493-854)$ \\
\hline
\end{tabular}

Categorical variables are displayed as the number (\%) of patients. Continuous variables are displayed as the mean $\pm \mathrm{SD}$ or as median (IQR).

The median preoperative GH level was $11.1 \mathrm{ng} / \mathrm{ml}$ (IQR 3.2-37.1 ng/ml), and the median preoperative IGF-1 level was $576 \mathrm{ng} / \mathrm{ml}$ (IQR 493-854 ng/ml) (Table 1). 


\section{Preoperative Radiological and Endocrinological Tumor Features}

The median tumor diameter at presentation was $1.6 \mathrm{~cm}$ (IQR 1.1-2.3 cm); 10 lesions (19\%) were microadenomas, and $42(81 \%)$ were macroadenomas. Preoperative MRI demonstrated suprasellar extension in 18 patients $(35 \%)$, infrasellar invasion in 40 patients (77\%), and cavernous sinus invasion in 7 patients (13\%). Using standard Knosp criteria, 18 tumors (35\%) exhibited a Knosp score of 0, 13 $(25 \%)$ presented with a score of $1,10(19 \%)$ were given a score of 2, $4(8 \%)$ exhibited a score of $3 \mathrm{~A}$, and $3(6 \%)$ were given a score of 4 . Six tumors $(11 \%)$ were purely intrasellar on preoperative MRI (Table 2). Three patients (6\%) had preoperative MR images obtained at outside institutions that were unavailable for review, and $1(2 \%)$ had a noncontrast preoperative MRI study that could not be reliably analyzed for invasion.

Invasive tumors had a significantly larger maximal diameter $(2.1 \mathrm{~cm}$ vs $0.62 \mathrm{~cm}$; p < 0.001, Mann-Whitney U-test). Preoperative GH and IGF-1 levels were both positively correlated with tumor size ( $p=0.003$, Spearman's rho and $\mathrm{p}=0.001$, Spearman's rho, respectively).

\section{Adenoma Pathology and Immunostaining}

All 52 patients had pathologically confirmed pituitary adenomas based on IHC. Forty-eight lesions (92\%) stained positive for $\mathrm{GH}, 30(58 \%)$ for prolactin, 13 (25\%) for thyroid-stimulating hormone (TSH), 4 (8\%) for adrenocorticotropic hormone, and 1 (2\%) for luteinizing hormone. The majority of lesions $(n=45,87 \%)$ were determined to be pure somatotroph adenomas. Five patients $(10 \%)$ were diagnosed with mammosomatotroph adenomas, and 1 (2\%) was diagnosed with a TSH- and prolactin-secreting adenoma with concurrent elevations in serum IGF-1 levels (preoperative IGF-1 = 505 ng/ml) (Table 2). The final patient $(2 \%)$ was diagnosed with a nonfunctional adenoma on pathological examination despite acromegalic clinical features and elevated IGF-1 levels (preoperative IGF-1 = $591 \mathrm{ng} / \mathrm{ml})$.

IHC staining data were not available for 3 patients (8\%). In 2 patients (4\%), pathology reports documenting IHC staining were unavailable. In one (2\%), a tissue sample large enough for pathological analysis could not be obtained during surgery. Clinical signs of acromegaly and elevated IGF-1 levels were observed in all of these patients despite the lack of IHC confirmation. Two patients (4\%) experienced hormonal remission in the postoperative period. The third patient (2\%) was lost to follow-up but exhibited a postoperative decrease in IGF-1 levels.

No significant correlations were found between pituitary adenoma subtype and tumor size, invasiveness, or hormonal remission.

\section{Postoperative Complications}

Intraoperative CSF leaks occurred in 14 cases (27\%). The majority of patients experienced no postoperative complications ( 35 patients, 67\%), with the most common complications being hyponatremia (9 patients, 17\%) and transient diabetes insipidus (DI) (3 patients, 6\%). Five $(10 \%)$ of the patients with hyponatremia were readmitted
TABLE 2. Tumor characteristics and pathology

\begin{tabular}{|c|c|}
\hline Tumor Characteristics \& Pathology & Value \\
\hline \multicolumn{2}{|l|}{ Tumor characteristics } \\
\hline Max median tumor size (IQR), cm & $1.6(1.1-2.3)$ \\
\hline Macroadenoma & $42(81)$ \\
\hline Microadenoma & $10(19)$ \\
\hline Suprasellar invasion & $18(35)$ \\
\hline Infrasellar extension & $40(77)$ \\
\hline Cavernous sinus invasion & $7(13)$ \\
\hline \multicolumn{2}{|l|}{ Knosp score } \\
\hline 0 & $18(35)$ \\
\hline 1 & $13(25)$ \\
\hline 2 & $10(19)$ \\
\hline $3 \mathrm{~A}$ & $4(8)$ \\
\hline $3 B$ & 0 \\
\hline 4 & $3(6)$ \\
\hline \multicolumn{2}{|l|}{ Immunohistochemical staining } \\
\hline $\mathrm{GH}$ & $48(92)$ \\
\hline Prolactin & $30(58)$ \\
\hline TSH & $13(25)$ \\
\hline Alpha subunit & $17(33)$ \\
\hline ACTH & $4(8)$ \\
\hline Luteinizing hormone & $1(2)$ \\
\hline \multicolumn{2}{|l|}{ Pituitary adenoma subtype } \\
\hline GH secreting & $45(87)$ \\
\hline Mammosomatotroph & $5(10)$ \\
\hline TSH + prolactin secreting & $1(2)$ \\
\hline Null-cell adenoma & $1(2)$ \\
\hline
\end{tabular}

ACTH = adenocorticotropic hormone.

Aside from maximal median tumor size, values are presented as the number (\%) of patients.

and required intervention, and it self-resolved in the other $4(8 \%)$. Two of the 3 patients $(4 \%)$ who displayed transient DI required no intervention, and the other patient (2\%) experienced symptom resolution prior to postoperative discharge after administration of one dose of desmopressin. A comprehensive list of postoperative complications can be found in Table 3. There were no correlations between tumor invasiveness or Knosp score and postoperative complications. One patient (2\%) was diagnosed postoperatively with new panhypopituitarism and required hydrocortisone, levothyroxine, and estradiol hormone replacement.

\section{Surgical Outcomes}

Of the 52 patients, $36(69 \%)$ underwent gross-total resection (GTR), and 16 (31\%) underwent subtotal resection (STR) (Table 3). The mean neuroimaging follow-up duration was $21.7 \pm 18.4$ months (Table 1).

Smaller tumors were more likely to be completely resected $(\mathrm{GTR}=1.47 \mathrm{~cm}$ vs $\mathrm{STR}=2.74 \mathrm{~cm} ; \mathrm{p}=0.002$, independent-samples t-test). A GTR was much less likely to occur when tumors invaded the infrasellar space or the cavernous sinuses ( $p=0.044$, Fisher's exact test; $p=$ 
TABLE 3. Postoperative surgical and endocrinological outcomes

\begin{tabular}{|c|c|}
\hline Outcome & Value \\
\hline \multicolumn{2}{|l|}{ Postop complication } \\
\hline Hyponatremia & $9(17)$ \\
\hline Transient DI & $3(6)$ \\
\hline Epistaxis & $4(8)$ \\
\hline CSF leak & $2(4)$ \\
\hline Permanent DI & $1(2)$ \\
\hline Meningitis & $1(2)$ \\
\hline Bacteremia/sepsis & $1(2)$ \\
\hline None & $35(67)$ \\
\hline \multicolumn{2}{|l|}{ EOR } \\
\hline GTR & $36(69)$ \\
\hline STR & $16(31)$ \\
\hline \multicolumn{2}{|l|}{ Endocrinological remission } \\
\hline Yes & $45(87)$ \\
\hline No & $7(14)$ \\
\hline Time to remission, median (IQR), mos & $3.0(4.25)$ \\
\hline \multicolumn{2}{|l|}{ Lab value, median (IQR), ng/ml } \\
\hline POD1 GH & $1.65(0.84-3.6)$ \\
\hline Most recent follow-up IGF-1 & $216(140-263)$ \\
\hline Follow-up IGF-1 apex & $252(209-360)$ \\
\hline Follow-up IGF-1 nadir & $195(127-253)$ \\
\hline \multicolumn{2}{|l|}{ Additional treatment } \\
\hline Medical therapy & $12(23)$ \\
\hline External radiotherapy & $2(4)$ \\
\hline Repeat resection & $1(2)$ \\
\hline None & $39(75)$ \\
\hline
\end{tabular}

Values are presented as the number (\%) of patients unless otherwise indicated.

0.026, Pearson chi-square test). One-way ANOVA showed that Knosp scores were significantly correlated with EOR, with increasing scores leading to lower GTR rates $(\mathrm{F}(4,43)$ $=4.88, \mathrm{p}=0.002$ )

\section{Adjuvant Medical, Surgical, and Radiation Treatment}

Postoperatively, 12 patients (23\%) received additional treatment. Of these, 2 with mammosomatotrophs (4\%) were placed on a combined cabergoline plus SRL therapy. One of these patients (2\%) was prescribed Sandostatin Depot, and the other (2\%) was placed on lanreotide (Fig. 1). Three patients $(6 \%)$ were placed on cabergoline alone, $4(8 \%)$ were prescribed Sandostatin Depot, and $1(2 \%)$ was placed on lanreotide (Fig. 2).

One patient (2\%) with a mammosomatotroph adenoma underwent repeat transsphenoidal resection 5 months after the initial procedure. Due to remaining residual tumor, the patient was subsequently placed on cabergoline and Sandostatin Depot. The patient's disease has remained refractory despite adjuvant medical therapy.

One patient (2\%) with an unresectable residual somaotroph adenoma in the right cavernous sinus received 1 month of adjuvant radiation therapy (50 Gy in 25 frac-
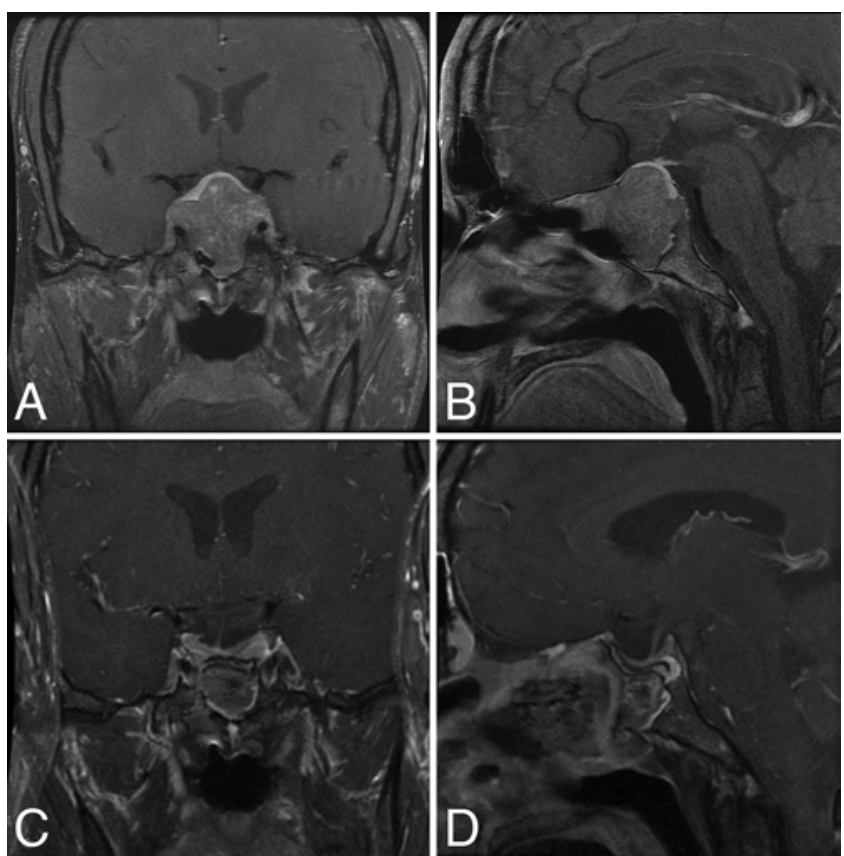

FIG. 1. Preoperative (A and B) and postoperative (C and D) coronal (left) and sagittal (right) postcontrast T1-weighted MR images demonstrating an invasive mammosomatotroph in a female patient presenting with signs and symptoms of acromegaly. Note the suprasellar extension, bilateral cavernous sinus invasion, and extensive bony invasion, including the tuberculum, planum sphenoidale, clivus, and sellar floor, with residual pituitary gland draped over the tumor with anterosuperior displacement of her infundibulum. The patient underwent near-total resection of her invasive adenoma, which stained positively for prolactin and $\mathrm{GH}$, and has been in remission with the addition of adjuvant cabergoline and lanreotide medical therapy to manage her small residual disease.

tions) that was well tolerated but did not result in significant decrease in tumor size. This patient was one of the aforementioned patients who were prescribed Sandostatin Depot, but her acromegaly has continued to be refractory to treatment. Another patient $(2 \%)$ with unresectable residual TSH- and prolactin-secreting adenoma in the right cavernous sinus received 1 month of adjuvant radiation therapy (180 Gy in 27 fractions) that was well tolerated and resulted in a decrease in tumor size. This patient is one of the aforementioned patients who is on postoperative cabergoline monotherapy and is in remission.

Figure 3 outlines postoperative outcomes and adjuvant treatment for patients in this study.

\section{Clinical Outcomes}

Of the 21 patients $(40 \%)$ who presented with headache, $16(76 \%)$ reported improvement postoperatively with 5 (24\%) reporting no change. Four (57\%) of the 7 patients who presented with preoperative visual changes reported improvement postoperatively, with 3 (43\%) reporting no change.

\section{Endocrinological Outcomes}

The median morning POD1 GH level was $1.65 \mathrm{ng} / \mathrm{ml}$ (IQR 0.84-3.6 ng/ml). The mean clinical follow-up time was $24.4 \pm 19.1$ months (Table 1$)$. The last known me- 

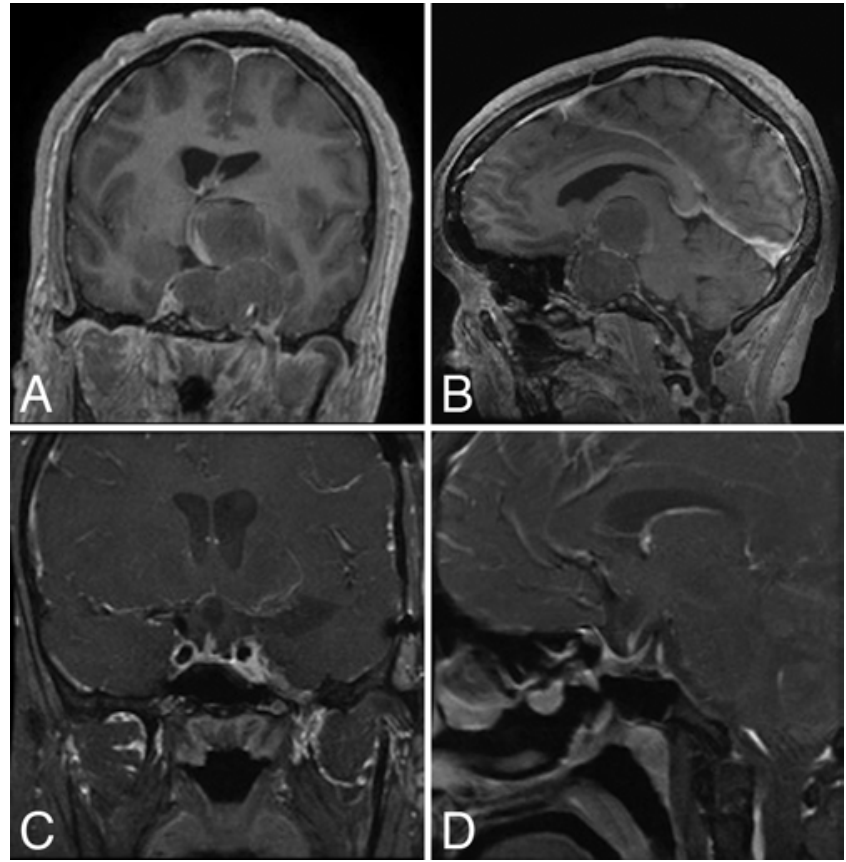

FIG. 2. Preoperative (A and $B$ ) and postoperative ( $C$ and $D)$ coronal (left) and sagittal (right) postcontrast T1-weighted MR images demonstrating a giant somatotroph in a young woman who suffered from features of advanced acromegaly. She also suffered from vision loss and headaches consistent with apoplexy, with patchy areas of contrast enhancement and intrinsic T1 signal change (not shown) consistent with acute hemorrhage. Her tumor demonstrated Knosp grade 4 invasion of her left cavernous sinus, with suprasellar extension, including a large nodule placing mass effect on her thalamus, third ventricle, hypothalamus, and mammillary bodies. The tumor additionally invaded the clivus and sellar floor, with obliteration of the patient's sphenoid sinus. The patient underwent a two-stage open craniotomy and endoscopic endonasal transsphenoidal resection of her adenoma for a near-total resection. Following an initial drop in her IGF-1 levels, she achieved full biochemical remission with the use of monthly Sandostatin Depot.

dian IGF-1 level (as measured at most recent follow-up at a mean of $24.4 \pm 19.1$ months) was $216 \mathrm{ng} / \mathrm{ml}$ (IQR 140-263 $\mathrm{ng} / \mathrm{ml}$ ) (Table 3).

Hormonal remission occurred in 45 patients $(87 \%)$, with a median time to remission of 3.0 months (IQR 4.25 months). Thirty-three patients (63\%) exhibited normalized IGF-1 levels within 4 months postoperatively. Four patients $(8 \%)$ did not return for follow-up within the first 4 months, but laboratory studies demonstrated normalized IGF-1 levels during their first postoperative visit (at 5, 6, 8 , and 42 months, respectively). Seven (13\%) of the other 8 patients experienced remission after initiation of postoperative medical therapy. The last patient $(2 \%)$ declined adjuvant medical therapy and exhibited normalized IGF-1 levels 29 months postoperatively without additional treatment. Eighty-six percent of patients with macroadenomas and $90 \%$ of those with microadenomas experienced postoperative remission. None of the patients included in this series presented with recurrence of disease after hormonal remission was achieved.

In order to accurately assess predictors of hormonal remission, patients who exhibited remission after postoper- ative medical therapy were analyzed separately (see next section). In patients who experienced remission from surgery alone, normalization of IGF-1 levels was more likely to occur in patients with smaller tumors $(p=0.036$, independent-samples t-test) and those who underwent GTR ( $\mathrm{p}<0.001$, Fisher's exact test). One-way ANOVA demonstrated that increasing Knosp scores were correlated with decreased rates of hormonal remission $(\mathrm{F}(4,36)=3.3$, $\mathrm{p}=$ $0.02)$. Patients with lower preoperative IGF-1 levels $(\mathrm{p}=$ 0.023 , Mann-Whitney U-test) and lower POD1 GH levels $(\mathrm{p}=0.013$, Mann-Whitney U-test) were also more likely to experience hormonal remission. The mean preoperative IGF-1 levels were $580 \pm 256 \mathrm{ng} / \mathrm{ml}$ in patients who remitted postoperatively versus $820 \pm 280 \mathrm{ng} / \mathrm{ml}$ in those who did not (Fig. 4).

\section{Hormonal Remission After Postoperative Medical Therapy}

Seven patients (13\%) experienced delayed biochemical remission after failing to achieve age- and sex-normalized IGF-1 levels at their first postoperative follow-up (6 weeks). All were prescribed medications to treat their residual disease at this time. Five (10\%) were placed on SRL therapy alone, and 2 (2\%) received only cabergoline. Following induction of medical therapy, the mean time to remission in these patients was 7.5 months, and all remitted within 12 months postoperatively.

These patients all had significantly larger tumors $(\mathrm{p}<$ 0.001 , Mann-Whitney U-test) and were less likely to undergo GTR ( $p=0.008$, Fisher's exact test) compared to patients who showed biochemical remission at their first postoperative follow-up. They also all exhibited significantly higher POD1 GH ( $p<0.001$, Mann-Whitney U-test) and preoperative IGF-1 ( $\mathrm{p}=0.001$, independent-samples t-test) levels than patients who remitted immediately postoperatively without adjuvant medical therapy.

\section{Differences in Outcomes by Hospital}

Eighteen patients $(35 \%)$ underwent surgical resection of their pituitary adenoma at LAC + USC Medical Center. The other 34 patients (65\%) underwent surgery at Keck Hospital, a private hospital affiliated with the Keck School of Medicine of USC. LAC + USC patients were significantly less likely to experience hormonal remission than Keck patients ( $\mathrm{p}=0.041$, Fisher's exact test). However, no significant correlation was observed between tumor size, invasiveness, or EOR and hospital. There was also no significant difference in mean preoperative IGF-1 levels or POD1 GH levels and the hospital at which the patient underwent surgery.

\section{Discussion}

In this study we analyzed the clinical and surgical outcomes of 52 patients with pituitary adenomas who met the diagnostic criteria for acromegaly and subsequently underwent endoscopic transsphenoidal resection of their tumors. The mean age of patients was $50.7 \pm 13.4$ years, and the male/female ratio was 1:2.25. Our study size was comparable to similar series on acromegaly, $, 5,26,27$ with our patients being older and with more being female.,10,21,28 $\mathrm{We}$ found that resection alone was sufficient to lead to hor- 


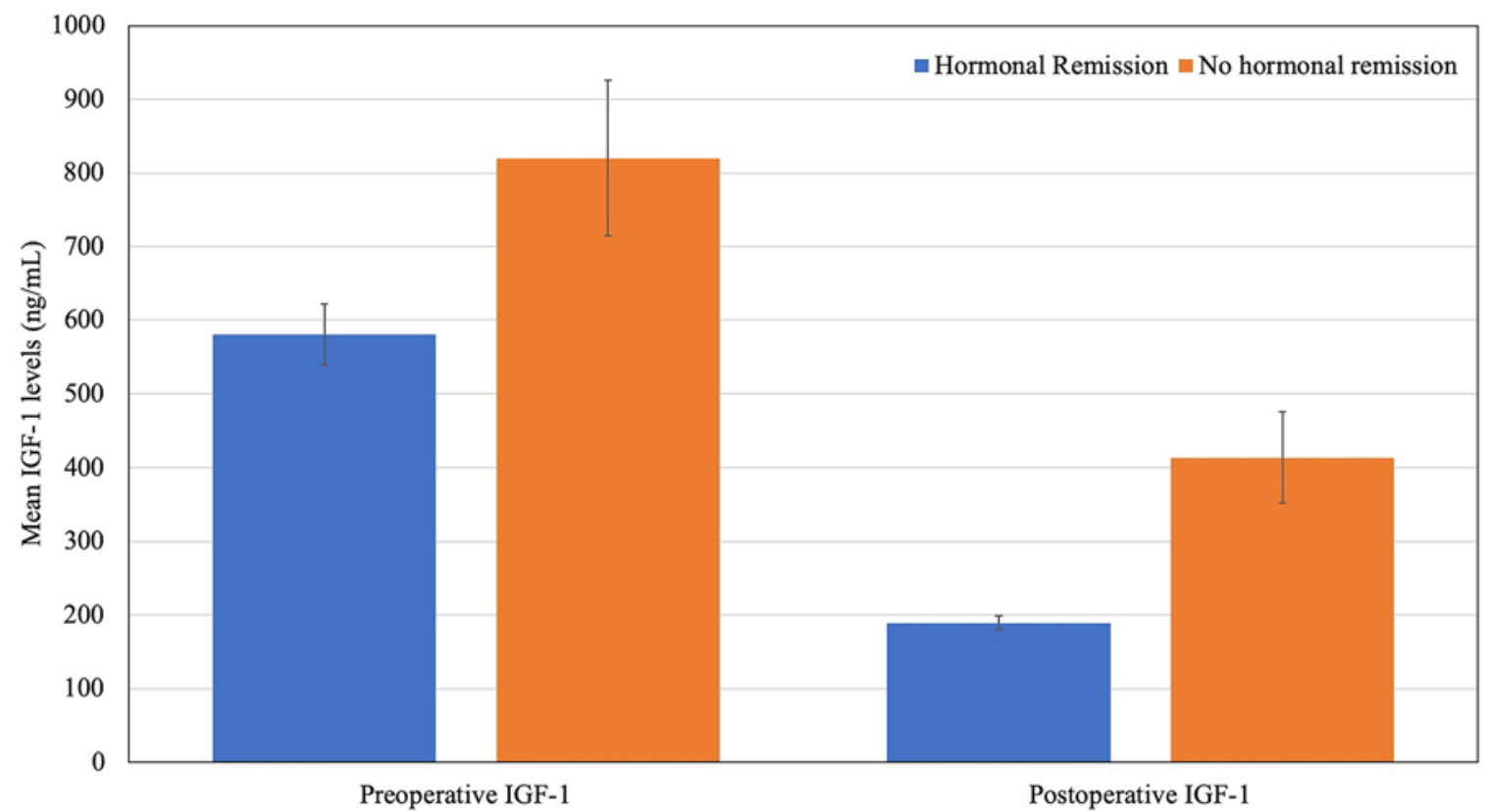

FIG. 3. Flowchart depicting postoperative outcomes and additional treatments for patients included in this series.

monal remission, as defined by age- and sex-normalized IGF-1 levels, in $73 \%$ of all patients. Adjuvant medical therapy increased the biochemical remission rate to $87 \%$. While some studies have demonstrated remission rates below 50\%, 21,25,26 a recent meta-analysis showed a long-term remission rate of $73 \%$ following endoscopic transsphenoidal resection. ${ }^{3}$
The use of the endoscopic endonasal approach (EEA) for resection of GH-secreting pituitary adenomas is supported by literature demonstrating no differences in patient outcomes between microsurgical treatment and EEA for the management of acromegaly. ${ }^{3,18}$ Additionally, resection of somatotroph adenomas via EEA has been shown to be associated with shorter operative time, as well as

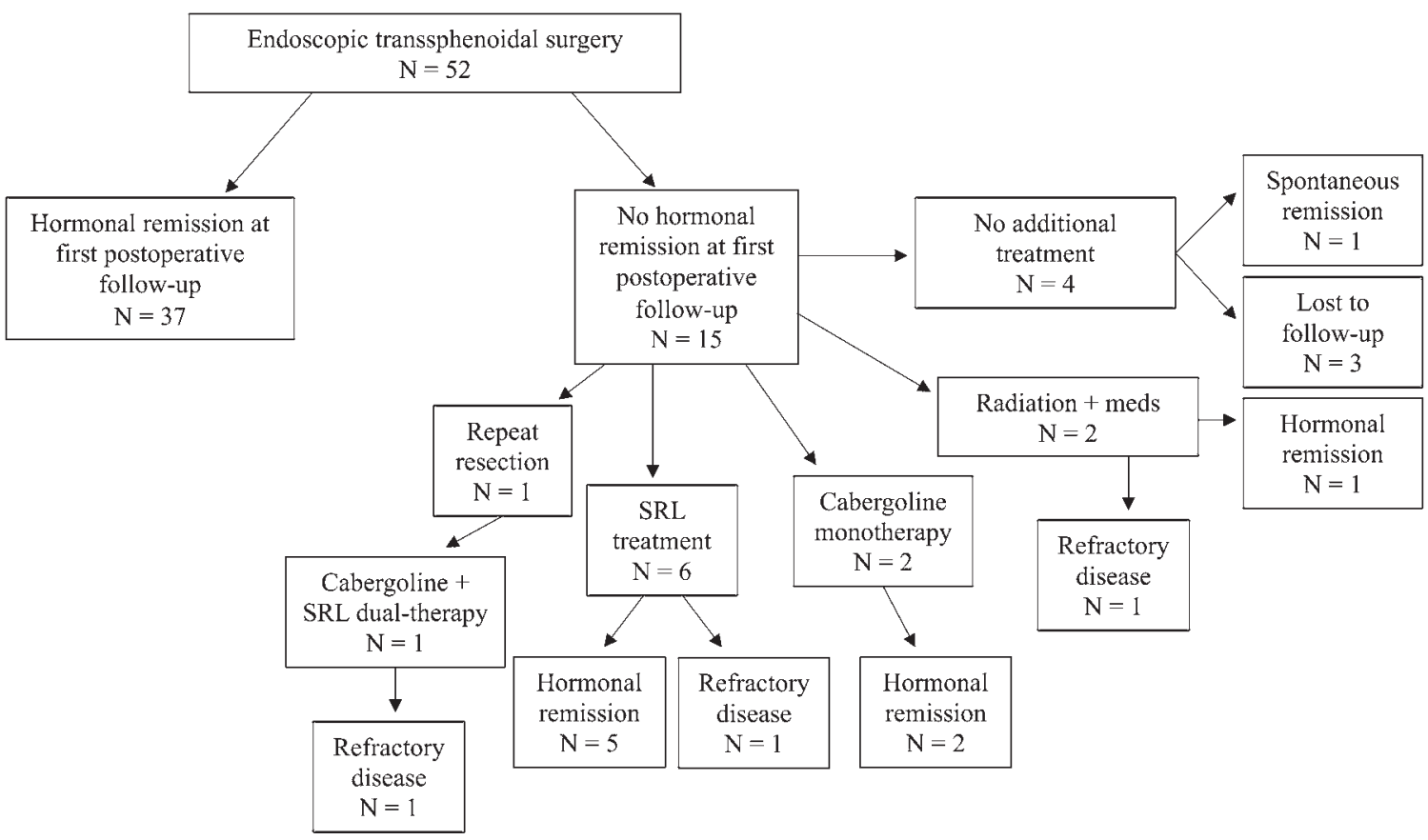

FIG. 4. In patients who experienced biochemical remission after surgery alone, preoperative IGF-1 levels were predictive of postoperative remission, with patients who presented with lower IGF-1 levels having a higher likelihood of remission postoperatively ( $p$ $=0.023$, Mann-Whitney U-test). The mean preoperative IGF-1 level was $580 \pm 256 \mathrm{ng} / \mathrm{ml}$ in patients who experienced remission postoperatively versus $820 \pm 280 \mathrm{ng} / \mathrm{ml}$ in those who did not. 
decreased blood loss, pain, infection rate, and length of hospital stay. ${ }^{3,18}$ Not only did patients included in this study experience a low rate of postoperative complications, but they also exhibited a higher than average rate of postoperative hormonal remission. Our postoperative complication rate was comparable to the overall rate found by Chen et al. ${ }^{3}$ in a recent systematic review of acromegalic patients treated with resection, with the most frequent complications (intraoperative CSF leakage, hyponatremia, and transient DI) being reported in similar frequencies in the literature. ${ }^{6,12,26,28}$

The current literature indicates that tumor size and invasiveness are important predictors of postoperative hormonal remission in patients with acromegaly. Specifically, residual tumor is commonly documented postoperatively in cases of pituitary adenomas that invade the cavernous sinuses. ${ }^{1,27,9,11,25}$ Jane et al. observed a correlation between increasing Knosp scores and hormonal remission in acromegalic patients..$^{2}$ Our patients presented with a comparable distribution of micro- and macroadenomas, as well as invasion patterns comparable to those of similar studies. ${ }^{8,11,18,21,28}$ We also found that tumor size, Knosp score, and EOR were significantly correlated with biological remission. Additionally, we enjoyed a higher rate of hormonal remission in patients with macroadenomas in this series (86\%) compared to other studies., ${ }^{1,12,21}$ This was likely due to the higher rate of GTR $(62 \%)$ we achieved in patients with macroadenomas compared with other studies. ${ }^{1}$

Numerous studies have shown preoperative GH level to be predictive of postoperative biochemical remission, $1,11,17$, $21,24,25$ but due to the pulsatile nature of its secretion, it is not our institutional practice to routinely gather preoperative $\mathrm{GH}$ levels in our patients. As a result, few preoperative $\mathrm{GH}$ levels were available for analysis and were not significantly correlated with hormonal remission. We did, however, observe a significant correlation between preoperative IGF-1 levels and postoperative remission, which has been reported elsewhere. ${ }^{9,12}$ The current literature also supports POD1 GH levels as a predictor of postoperative hormonal remission, and our results agreed with this as well.1,3,12

Postoperative adjuvant medical therapy is an important component of treating patients with acromegaly, particularly in those whose tumors cannot be fully resected due to proximity to one of the internal carotid arteries or invasion deep into one of the cavernous sinuses. While it is not within the guidelines to initiate treatment of residual disease until 12 weeks postoperatively, ${ }^{15}$ it is our institutional practice to begin treatment and then attempt weaning the patient off medication once normalization of the IGF-1 levels is achieved.

We were especially interested in comparing patient outcomes from LAC + USC Medical Center versus Keck Hospital of USC. Our results showed that county patients were significantly less likely to experience postoperative biochemical remission. As there were no significant differences in other predictive factors such as preoperative IGF1 or GH levels, tumor size, tumor invasiveness, EOR, or POD1 GH levels, we believe this difference is most likely due to an inherent tumor characteristic that is not captured by our data. One hypothesis is that these patients present later when their disease is at a more advanced stage. An- other possibility is that their tumors are more frequently atypical adenomas, which have been shown to be more aggressive and have hormonal hypersecretory features. ${ }^{20}$ Atypical adenomas are typically defined by MIB-1 (Ki67) indices and p53 staining; $;{ }^{20}$ however, due to the limited pathological analysis performed for patients operated on at LAC + USC, these data were not available for analysis.

All patients were included in this study because of clinical and laboratory findings indicative of acromegaly and suspicion of underlying GH secretion even in the absence of pathological confirmation. Immunostaining data were unavailable for 3 of the patients included in this series, and in another patient pathological analysis demonstrated a null-cell adenoma. While it is unusual for a suspected GH-secreting adenoma to not stain for GH, Schroeder et al. demonstrated that $3.7 \%$ of adenomas from clinically diagnosed acromegalic patients did not stain for GH in the setting of repeated testing. ${ }^{22}$ Furthermore, their study concluded that clinical and biochemical features of acromegaly are sufficient for diagnosis even in the absence of pathological verification, which validates our inclusion of these 4 patients. The existence of somatotroph-induced acromegaly in these patients is supported by the normalization of IGF-1 levels in 3 of the 4 patients following resection of their suspected GH-secreting adenomas. The fourth patient was lost to follow-up but exhibited decreased IGF-1 levels at the 5-month postoperative visit.

\section{Limitations}

This study was limited by its retrospective analysis and comparatively shorter follow-up duration. Analysis of long-term outcomes was also limited by patients who were lost to follow-up after their first postoperative clinic visit. Additionally, while our study is larger than many other purely endoscopic acromegaly series, the relatively small patient size may have limited our ability to achieve statistical significance when determining predictors of patient outcome. Other limitations include inconsistent measuring of preoperative $\mathrm{GH}$ levels and incomplete and inconsistent immunostaining of pituitary adenoma biopsy specimens. Per institutional policy, preoperative GH levels are not routinely acquired for the diagnosis of acromegaly in the setting of elevated IGF-1 levels and the presence of a pituitary adenoma detected on MRI. At LAC + USC Medical Center, immunostaining of pituitary adenomas is only performed for the suspected hormone(s) being secreted due to cost and assay availability. Similarly, routine testing for hormonal remission by GH suppression with OGTT is not performed in every patient but is reserved for equivocal cases with IGF-1 reduction not normalizing or rising during the postoperative timeframe.

\section{Conclusions}

In summary, we find that endoscopic transsphenoidal resection of GH-secreting pituitary adenomas can lead to hormonal remission in up to $87 \%$ of patients when combined with adjuvant medical therapy. Lower preoperative IGF-1 and POD1 GH levels, as well as greater EOR, were significantly correlated with a higher rate of postoperative hormonal remission. EOR was shown to be dependent on 
tumor size and invasiveness, where invasion into the cavernous sinuses and higher Knosp scores were associated with lower rates of resection. Additionally, patients with higher preoperative IGF-1 and GH levels were more likely to present with larger tumors. For patients in whom biochemical remission failed to be achieved by resection alone, treatment with SRL therapy is recommended. Further research is indicated to determine differences in patients who experience hormonal remission during SRL treatment and those who continue to exhibit high levels of IGF-1. The primary limitations of this study are its retrospective design and comparatively shorter overall follow-up duration.

\section{References}

1. Albarel F, Castinetti F, Morange I, Conte-Devolx B, Gaudart $\mathrm{J}$, Dufour H, et al: Outcome of multimodal therapy in operated acromegalic patients, a study in 115 patients. Clin Endocrinol (Oxf) 78:263-270, 2013

2. Campbell PG, Kenning E, Andrews DW, Yadla S, Rosen M, Evans JJ: Outcomes after a purely endoscopic transsphenoidal resection of growth hormone-secreting pituitary adenomas. Neurosurg Focus 29(4):E5, 2010

3. Chen CJ, Ironside N, Pomeraniec IJ, Chivukula S, Buell TJ, Ding D, et al: Microsurgical versus endoscopic transsphenoidal resection for acromegaly: a systematic review of outcomes and complications. Acta Neurochir (Wien) 159:2193-2207, 2017

4. Dusek T, Kastelan D, Melada A, Baretic M, Skoric Polovina T, Perkovic Z, et al: Clinical features and therapeutic outcomes of patients with acromegaly: single-center experience. J Endocrinol Invest 34:e382-e385, 2011

5. Fathalla H, Cusimano MD, Di Ieva A, Lee J, Alsharif O, Goguen J, et al: Endoscopic versus microscopic approach for surgical treatment of acromegaly. Neurosurg Rev 38:541549,2015

6. Freda PU, Wardlaw SL, Post KD: Long-term endocrinological follow-up evaluation in 115 patients who underwent transsphenoidal surgery for acromegaly. J Neurosurg 89:353358,1998

7. Giustina A, Chanson P, Bronstein MD, Klibanski A, Lamberts S, Casanueva FF, et al: A consensus on criteria for cure of acromegaly. J Clin Endocrinol Metab 95:3141-3148, 2010

8. Gondim JA, Almeida JP, de Albuquerque LA, Gomes E, Schops M, Ferraz T: Pure endoscopic transsphenoidal surgery for treatment of acromegaly: results of 67 cases treated in a pituitary center. Neurosurg Focus 29(4):E7, 2010

9. Haliloglu O, Kuruoglu E, Ozkaya HM, Keskin FE, Gunaldi $\mathrm{O}, \mathrm{Oz} \mathrm{B}$, et al: Multidisciplinary approach for acromegaly: a single tertiary center's experience. World Neurosurg 88:270-276, 2016

10. Hazer DB, Işık S, Berker D, Güler S, Gürlek A, Yücel T, et al: Treatment of acromegaly by endoscopic transsphenoidal surgery: surgical experience in 214 cases and cure rates according to current consensus criteria. J Neurosurg 119:1467-1477, 2013

11. Hofstetter CP, Mannaa RH, Mubita L, Anand VK, Kennedy JW, Dehdashti AR, et al: Endoscopic endonasal transsphenoidal surgery for growth hormone-secreting pituitary adenomas. Neurosurg Focus 29(4):E6, 2010

12. Jane JA Jr, Starke RM, Elzoghby MA, Reames DL, Payne SC, Thorner MO, et al: Endoscopic transsphenoidal surgery for acromegaly: remission using modern criteria, complications, and predictors of outcome. J Clin Endocrinol Metab 96:2732-2740, 2011

13. Katznelson L, Laws ER Jr, Melmed S, Molitch ME, Murad $\mathrm{MH}, \mathrm{Utz}$ A, et al: Acromegaly: an Endocrine Society clinical practice guideline. J Clin Endocrinol Metab 99:3933-3951, 2014

14. Melmed S: Acromegaly. N Engl J Med 322:966-977, 1990

15. Melmed S, Bronstein MD, Chanson P, Klibanski A, Casanueva FF, Wass JAH, et al: A consensus statement on acromegaly therapeutic outcomes. Nat Rev Endocrinol 14:552-561, 2018

16. O'Malley BW Jr, Grady MS, Gabel BC, Cohen MA, Heuer GG, Pisapia J, et al: Comparison of endoscopic and microscopic removal of pituitary adenomas: single-surgeon experience and the learning curve. Neurosurg Focus 25(6):E10, 2008

17. Parkinson C, Renehan AG, Ryder WD, O’Dwyer ST, Shalet SM, Trainer PJ: Gender and age influence the relationship between serum GH and IGF-I in patients with acromegaly. Clin Endocrinol (Oxf) 57:59-64, 2002

18. Phan K, Xu J, Reddy R, Kalakoti P, Nanda A, Fairhall J: Endoscopic endonasal versus microsurgical transsphenoidal approach for growth hormone-secreting pituitary adenomas - systematic review and meta-analysis. World Neurosurg 97:398-406, 2017

19. Ribeiro-Oliveira A Jr, Barkan A: The changing face of acromegaly-advances in diagnosis and treatment. Nat Rev Endocrinol 8:605-611, 2012

20. Rutkowski MJ, Alward RM, Chen R, Wagner J, Jahangiri A, Southwell DG, et al: Atypical pituitary adenoma: a clinicopathologic case series. J Neurosurg 128:1058-1065, 2018

21. Sarkar S, Rajaratnam S, Chacko G, Chacko AG: Endocrinological outcomes following endoscopic and microscopic transsphenoidal surgery in 113 patients with acromegaly. Clin Neurol Neurosurg 126:190-195, 2014

22. Schroeder JL, Spiotta AM, Fleseriu M, Prayson RA, Hamrahian $\mathrm{AH}$, Weil RJ: Absence of immunostaining for growth hormone in a subset of patients with acromegaly. Pituitary 17:103-108, 2014

23. Shen M, Tang Y, Shou X, Wang M, Zhang Q, Qiao N, et al: Surgical results and predictors of initial and delayed remission for growth hormone-secreting pituitary adenomas using the 2010 consensus criteria in 162 patients from a single center. World Neurosurg 124:e39-e50, 2019

24. Starke RM, Raper DM, Payne SC, Vance ML, Oldfield EH, Jane JA Jr: Endoscopic vs microsurgical transsphenoidal surgery for acromegaly: outcomes in a concurrent series of patients using modern criteria for remission. J Clin Endocrinol Metab 98:3190-3198, 2013

25. van Bunderen CC, van Varsseveld NC, Baayen JC, van Furth WR, Aliaga ES, Hazewinkel MJ, et al: Predictors of endoscopic transsphenoidal surgery outcome in acromegaly: patient and tumor characteristics evaluated by magnetic resonance imaging. Pituitary 16:158-167, 2013

26. Wagenmakers MA, Netea-Maier RT, van Lindert EJ, Pieters GF, Grotenhuis AJ, Hermus AR: Results of endoscopic transsphenoidal pituitary surgery in 40 patients with a growth hormone-secreting macroadenoma. Acta Neurochir (Wien) 153:1391-1399, 2011

27. Yildirim AE, Sahinoglu M, Divanlioglu D, Alagoz F, Gurcay AG, Daglioglu E, et al: Endoscopic endonasal transsphenoidal treatment for acromegaly: 2010 consensus criteria for remission and predictors of outcomes. Turk Neurosurg 24:906-912, 2014

28. Zhou T, Wang F, Meng X, Ba J, Wei S, Xu B: Outcome of endoscopic transsphenoidal surgery in combination with somatostatin analogues in patients with growth hormone producing pituitary adenoma. J Korean Neurosurg Soc 56:405-409, 2014

\section{Disclosures}

The authors report no conflict of interest concerning the materi- 
als or methods used in this study or the findings specified in this paper.

\section{Author Contributions}

Conception and design: Rutkowski, Carmichael, Zada. Acquisition of data: Cardinal, Shiroishi, Liu, Wrobel. Analysis and interpretation of data: Rutkowski, Cardinal, Micko, Shiroishi, Liu. Drafting the article: Rutkowski, Cardinal. Critically revising the article: Rutkowski, Carmichael, Zada. Reviewed submitted version of manuscript: Rutkowski, Carmichael, Zada. Statistical analysis: Cardinal. Administrative/technical/material support: Wrobel. Study supervision: Carmichael, Zada.

\section{Correspondence}

Martin J. Rutkowski: Keck School of Medicine of University of Southern California, Los Angeles, CA. martin.rutkowski@gmail. com. 